02

\title{
Зондовая спектроскопия комбинационного рассеяния света поликристаллов ураниловых соединений
}

\author{
(C) В.С. Горелик ${ }^{1,2}$, C.О. Нечипуренко ${ }^{1}$, А.А. Лобойко ${ }^{1}$ \\ ${ }^{1}$ Физический институт им. П.Н. Лебедева РАН, \\ 119991 Москва, Россия \\ ${ }^{2}$ МГТУ им. Н.Э. Баумана, \\ 105005 Москва, Россия \\ e-mail: gorelik@sci.lebedev.ru
}

Поступила в редакцию 22.05.2019 г.

В окончательной редакции 22.05.2019 г.

Принята к публикации 04.06.2019 г.

Исследованы закономерности в спектрах комбинационного рассеяния ацетатных ураниловых соединений $\mathrm{CsUO}_{2}\left(\mathrm{CH}_{3} \mathrm{COO}\right)_{3}, \mathrm{CsUO}_{2}\left(\mathrm{CD}_{3} \mathrm{COO}\right)_{3}, \mathrm{NaUO}_{2}\left(\mathrm{CH}_{3} \mathrm{COO}\right)_{3}, \mathrm{NaUO}_{2}\left(\mathrm{CD}_{3} \mathrm{COO}\right)_{3}, \mathrm{UO}_{2}\left(\mathrm{CH}_{3} \mathrm{COO}\right)_{2}$ и $\mathrm{RbUO}_{2}\left(\mathrm{CH}_{3} \mathrm{COO}\right)_{3}$. Возбуждение спектров комбинационного рассеяния осуществлялось лазерным излучением с длиной волны $785 \mathrm{~nm}$ и регистрировалось с помощью волоконно-оптического миниспектрометра с многоэлементным приемником. Установлено, что в спектрах всех этих соединений присутствует интенсивный спутник комбинационного рассеяния, частота которого находится в диапазоне $850-860 \mathrm{~cm}^{-1}$. Этот спутник соответствует проявлению симметричных валентных колебаний $\left(A_{1}\right.$-типа) ураниловой группы. Полученные результаты открывают возможность для обнаружения и анализа ураниловых соединений в небольших количествах.

Ключевые слова: уранил, уранилацетат, комбинационное рассеяние света, оптическое возбуждение, спектр, лазер.

DOI: $10.21883 /$ OS.2019.10.48353.164-19

\section{Введение}

Применение методов спектроскопии комбинационного рассеяния (КР) света широко используется для получения информации о молекулярной структуре различных твердотельных соединений. Важной практической задачей является регистрация спектров КР ураниловых соединений для их обнаружения в окружающей среде в связи с их эффективным использованием в промышленных целях. В ураниловых соединениях присутствуют молекулы, содержащие ион уранила $\left(\mathrm{UO}_{2}\right)^{+}$. Изолированная группа уранила имеет четыре нормальных колебания: полносимметричное валентное колебание $v_{s}$ вдоль оси $\mathrm{O}-\mathrm{U}-\mathrm{O}$, деформационное дважды вырожденное колебание $v_{2}$, при котором колебания атомов урана и кислорода происходят в противофазе в двух взаимно перпендикулярных плоскостях. Антисимметричное колебание $v_{a s}$ соответствует нарушению равноплечности колебаний вдоль оси уранила. Группы $\left(\mathrm{UO}_{2}\right)^{+}$ близки линейным и расположены вдоль оси точечной группы симметрии исследуемых кристаллов. Уранилы образует разнообразные комплексы с органическими и неорганическими лигандами. Как показывают расчеты и результаты экспериментальных исследований, частота полносимметричной валентной моды для свободного иона уранила близка к $850 \mathrm{~cm}^{-1}[1-3]$. Присутствие соответствующего комбинационного сателлита в спектрах КР анализируемых веществ может служить надежным индикатором иона уранила. Практическое использова- ние метода спектроскопии КР до последнего времени было затруднено [4-7] в связи с необходимостью использования громоздкого оборудования в виде двойного или тройного монохроматоров и высокочувствительного одноканального приемника излучения - фотоумножителя [8-10]. При этом удавалось осуществлять регистрацию спектра КР при большом времени экспозиции и для достаточно большого количества исследуемого вещества. В частности, спектры КР в соединениях, содержащих диоксид урана, ранее были зарегистрированы в работе [11] при возбуждении гелий-неоновым лазером с мощностью $10 \mathrm{~mW}$. При этом использовался двойной монохроматор, обеспечивающий разрешение спектра около $2 \mathrm{~cm}^{-1}$. В работе [12] исследовались спектры КР некоторых поликристаллов минералов, содержащих диоксид урана, с использованием двойного монохроматора и гелий-неонового лазера с мощностью $6 \mathrm{~mW}$. В КРспектрах, полученных в этих работах, наблюдался самый интенсивный пик в области $840-875 \mathrm{~cm}^{-1}$, соответствующий полносимметричной моде иона уранила. Спектры КР водных растворов уранилацетатов были получены в работе [13] при возбуждении гелий-неоновым лазером $(\lambda=632.8 \mathrm{~nm})$. В этих спектрах отчетливо проявлялись линии с частотами 841 и $928 \mathrm{~cm}^{-1}$. Эти линии соответствуют симметричным и антисимметричным колебаниям ураниловой группы.

Спектры твердотельных соединений на основе уранилацетата исследовались в работах $[6,14-16]$. В работе [6] был зарегистрирован спектр КР твердых кристаллов 
Пространственные группы симметрии исследуемых соединений

\begin{tabular}{c|l|c|c|c}
\hline № & Название соединения & Пространственная группа симметрии & Сингония & Центр инверсии \\
\hline 1 & $\mathrm{CsUO}_{2}\left(\mathrm{CH}_{3} \mathrm{COO}\right)_{3}$ & $D_{2 h}^{15}(P b c a)$ & Ромбическая & Присутствует \\
2 & $\mathrm{NaUO}_{2}\left(\mathrm{CH}_{3} \mathrm{COO}\right)_{3}$ & $T^{4}\left(P 2_{1} 3\right)$ & Кубическая & Отсутствует \\
3 & $\mathrm{RbUO}_{2}\left(\mathrm{CH}_{3} \mathrm{COO}\right)_{3}$ & $C_{4 h}^{6}\left(I 4_{1} / a\right)$ & Тетрагональная & Присутствует \\
4 & $\mathrm{UO}_{2}\left(\mathrm{CH}_{3} \mathrm{COO}\right)_{2}$ & $D_{2 h}^{12}(P n n m)$ & Ромбическая & Присутствует \\
5 & $\mathrm{CsUO}_{2}\left(\mathrm{CD}_{3} \mathrm{COO}\right)_{3}$ & $D_{2 h}^{15}(P b c a)$ & Ромбическая & Присутствует \\
6 & $\mathrm{NaUO}_{2}\left(\mathrm{CD}_{3} \mathrm{COO}\right)_{3}$ & $T^{4}\left(P 2_{1} 3\right)$ & Кубическая & Отсутствует
\end{tabular}

$\mathrm{Zn}\left(\mathrm{UO}_{2}\right)_{2}\left(\mathrm{CH}_{3} \mathrm{COO}\right)_{6} \cdot 7 \mathrm{H}_{2} \mathrm{O}$ при возбуждении гелийнеоновым лазером $(\lambda=632.8$ нм $)$. В полученном спектре симметричным и антисимметричным колебаниям соответствовали линии на частотах 847 и $932 \mathrm{~cm}^{-1}$. Спектры КР твердого кристалла $\mathrm{NaUO}_{2} \mathrm{CH}_{3}(\mathrm{COO})_{3}$ изучались в работе [14]. В этой работе для записи спектра использовался фотоумножитель и спектрограф, возбуждение осуществлялось аргоновым лазером $(\lambda=514.5 \mathrm{~nm})$. В этом спектре проявлялась интенсивная линия на частоте $865 \mathrm{~cm}^{-1}$, отнесенная к симметричным валентным колебаниям. В работе [15] был получен спектр ИК поглощения в кристаллах $\mathrm{RbUO}_{2}\left(\mathrm{CH}_{3} \mathrm{COO}\right)_{3}$ и $\mathrm{CsUO}_{2}\left(\mathrm{CH}_{3} \mathrm{COO}\right)_{3}$. Установлено, что симметричным и антисимметричным колебаниям в кристаллах рубидийуранилацетата соответствуют частоты 852 и $924 \mathrm{~cm}^{-1}$, а в кристаллах цезий-уранилацетата 842 и $920 \mathrm{~cm}^{-1}$. Спектры КР в этих соединениях ранее не регистрировались. В работе [16] были исследованы спектры КР твердотельного кристаллогидрата уранилацетата $\mathrm{UO}_{2}\left(\mathrm{CH}_{3} \mathrm{COO}\right)_{6} \cdot 2 \mathrm{H}_{2} \mathrm{O}$, в которых также проявились симметричные и антисимметричные моды с частотами 867 и $942 \mathrm{~cm}^{-1}$.

В данной работе была поставлена задача регистрации КР-спектров ряда ураниловых соединений, в состав которых входит уранилацетат.

\section{Структура и свойства симметрии исследуемых кристаллов}

Исследуемые образцы представляли собой микрокристаллические порошки желтого или зеленоватого цветов соединений: $\mathrm{CsUO}_{2}\left(\mathrm{CH}_{3} \mathrm{COO}\right)_{3}, \mathrm{NaUO}_{2}\left(\mathrm{CH}_{3} \mathrm{COO}\right)_{3}$, $\mathrm{RbUO}_{2}\left(\mathrm{CH}_{3} \mathrm{COO}\right)_{3}, \mathrm{UO}_{2}\left(\mathrm{CH}_{3} \mathrm{COO}\right)_{2}, \mathrm{CsUO}_{2}\left(\mathrm{CD}_{3} \mathrm{COO}\right)_{3}$ и $\mathrm{NaUO}_{2}\left(\mathrm{CD}_{3} \mathrm{COO}\right)_{3}$. В работах [17-20] проводились кристаллоструктурные исследования этих соединений. Соответствующие пространственные группы симметрии обсуждаемых кристаллов приведены в таблице.

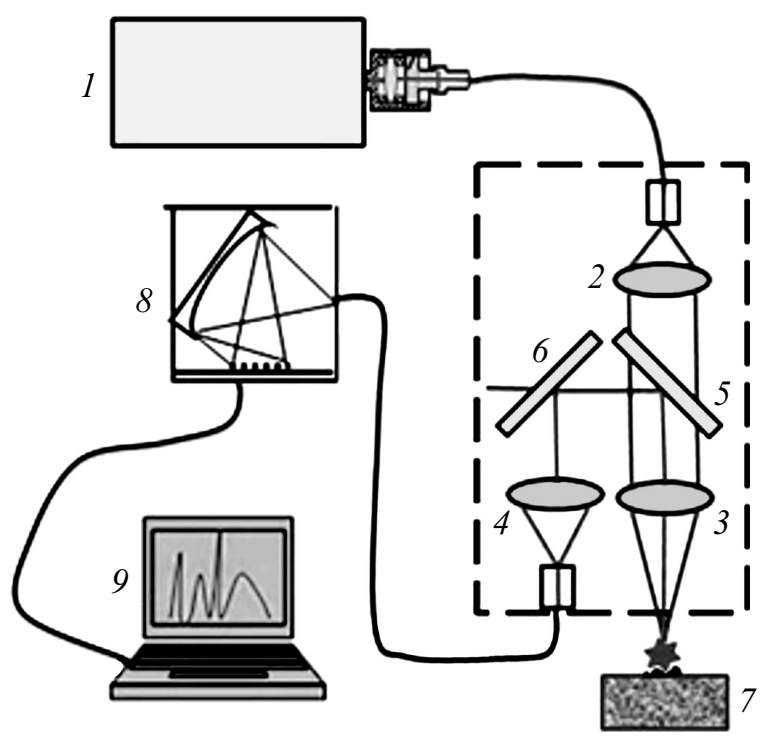

Pис. 1. Схема экспериментальной установки: 1 - лазерный источник излучения, 2, 3,4 - линзы, 5, 6 - интерференционные фильтры, 7 - предметный стол с образцом, 8 спектрометр, 9 - компьютер.

\section{Методика эксперимента}

Схема используемой экспериментальной установки приведена на рис. 1. Спектры КР исследуемых соединений регистрировались с помощью микрозондового волоконно-оптического миниспектрометра [21]. Возбуждение спектров КР осуществлялось с использованием непрерывного лазера с длиной волны $785 \mathrm{~nm}$ и средней мощностью $300 \mathrm{~mW}$. Разрешение при регистрации спектров КР составляло $2 \mathrm{~cm}^{-1}$. Чувствительность установки позволяла исследовать образцы с массой до сотой доли микрограмма. Излучение от лазера 1 с помощью кварцевых световодов подводилось к зонду и фокусировалось линзами 2,3 на исследуемый образец 7. Для подавления возбуждающего излучения использовались элементы 5, 6. Рассеянное излучение попадало на интерференционный фильтр 5 , который пропускал лазерное излучение, возбуждающее КР, и 

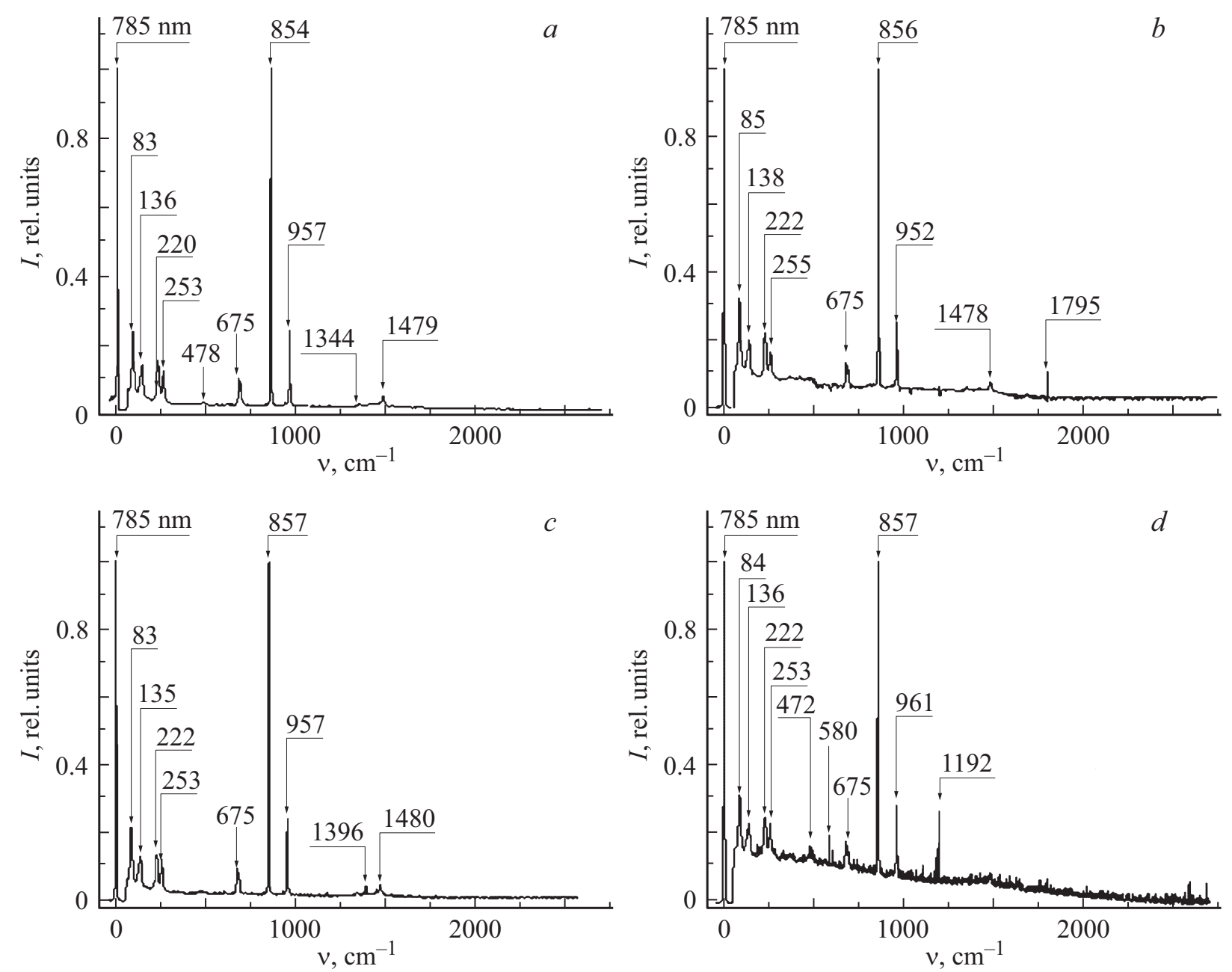

Рис. 2. Вид спектров КР в ураниловых соединениях: $a-\mathrm{CsUO}_{2}\left(\mathrm{CH}_{3} \mathrm{COO}\right)_{3}, b-\mathrm{NaUO}_{2}\left(\mathrm{CH}_{3} \mathrm{COO}\right)_{3}, c-\mathrm{RbUO}_{2}\left(\mathrm{CH}{ }_{3} \mathrm{COO}\right)_{3}$, $d-\mathrm{UO}_{2}\left(\mathrm{CH}_{3} \mathrm{COO}\right)_{2}$.

отражал длинноволновое излучение. При помощи отражающего зеркала 6 и линзы 4 рассеянное излучение подводилось к спектрометру 8 и затем анализировалось с помощью компьютера 9. Использование инфракрасного излучения с длиной волны $785 \mathrm{~nm}$ для возбуждения спектров КР обеспечивало подавление фотолюминесценции в исследуемых образцах, сильно люминесцирующих под действием коротковолнового излучения.

Возбуждающее излучение при фокусировке короткофокусной линзой проникало на небольшую глубину образца $\left(\sim 10^{-5} \mathrm{~cm}\right)$, при этом площадь поверхности образца, освещаемой возбуждающим излучением, составляла $\sim 10^{-4} \mathrm{~cm}^{2}$. Таким образом, предельно малый объем исследуемого вещества составлял $\sim 10^{-9} \mathrm{~cm}^{3}$. Плотность мощности сфокусированного излучения составляла $\sim 0.3 \mathrm{MW} / \mathrm{cm}^{2}$. Такая плотность мощности обеспечивала достаточно сильный сигнал КР при фокусировке лазерного излучения на исследуемом веществе. Существенного нагревания образца не происходило, так как длина волны возбуждающего излучения $(785 \mathrm{~nm})$ соответствует области прозрачности ацетатных ураниловых соединений.

\section{Результаты исследований и их обсуждение}

На рис. 2 приведены полученные спектры КР исследуемых водородосодержащих уранилацетатов: $\mathrm{CsUO}_{2}\left(\mathrm{CH}_{3} \mathrm{COO}\right)_{3} \quad(a), \quad \mathrm{NaUO}_{2}\left(\mathrm{CH}_{3} \mathrm{COO}\right)_{3} \quad(b)$, $\mathrm{RbUO}_{2}\left(\mathrm{CH}_{3} \mathrm{COO}\right)_{3}(c), \mathrm{UO}_{2}\left(\mathrm{CH}_{3} \mathrm{COO}\right)_{2}(d)$. На этом рисунке в спектрах КР наблюдается 10 максимумов интенсивности КР. Так, две низкочастотных линии с частотами, находящимися в диапазоне $80-140 \mathrm{~cm}^{-1}$, характерны для трансляционных колебаний радикальных групп ацетатов, а линии в диапазоне $220-250 \mathrm{~cm}^{-1}$ можно отнести к вибронным колебаниям. Самая интенсивная линия, лежащая в диапазоне $850-860 \mathrm{~cm}^{-1}$, соответствует полносимметричным колебаниям $v_{s}$ $(\mathrm{O}-\mathrm{U}-\mathrm{O})$. Линия с частотами $950-960 \mathrm{~cm}^{-1}$ относится 

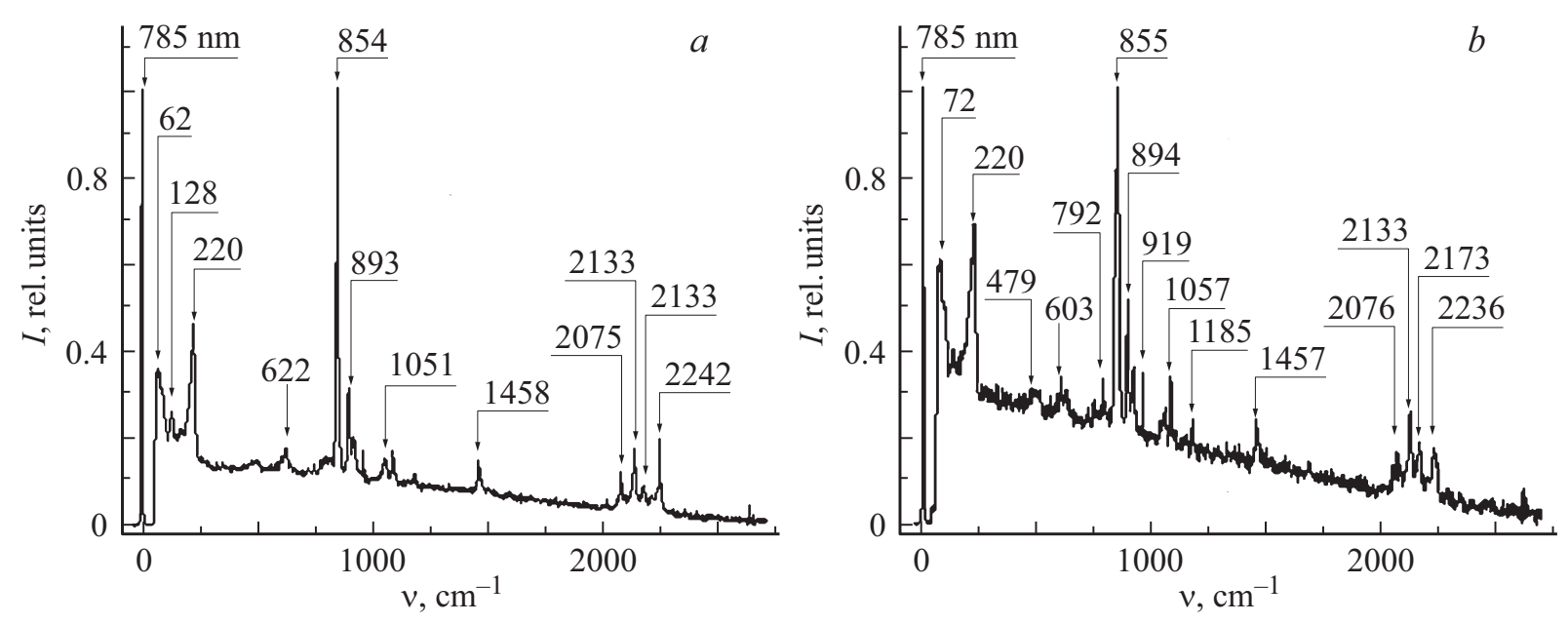

Рис. 3. Вид спектров КР в ураниловых соединениях: $a-\mathrm{CsUO}_{2}\left(\mathrm{CD}_{3} \mathrm{COO}\right)_{3}, b-\mathrm{NaUO}_{2}\left(\mathrm{CD}_{3} \mathrm{COO}\right)_{3}$.

к антисимметричной моде $v_{a s}$. Линии, находящиеся на частотах около 675 и $1480 \mathrm{~cm}^{-1}$, соответствуют колебаниям $\mathrm{O}-\mathrm{C}-\mathrm{O}$. Пик с частотой $1344 \mathrm{~cm}^{-1}$ (рис. 2,a) соответствует деформационным колебаниям группы $\mathrm{CH}_{3}$.

На рис. 3 приведены спектры КР соединений $\mathrm{CsUO}_{2}\left(\mathrm{CD}_{3} \mathrm{COO}\right)_{3}(a), \mathrm{NaUO}_{2}\left(\mathrm{CD}_{3} \mathrm{COO}\right)_{3}(b)$. Полосы на частотах $60-120 \mathrm{~cm}^{-1}$ соответствуют трансляционным модам ацетатных групп, эти моды сдвинуты в область низких частот в сравнении с недейтерированными соединениями (рис. 2). Линии с частотой $220 \mathrm{~cm}^{-1}$ соответствуют вибронным колебаниям ацетатных групп. Линии, находящиеся на частотах в диапазонах 600-620, $1050-1060$ и $1457-1458 \mathrm{~cm}^{-1}$, соответствуют колебаниям группы O-C-O. Полосы на частотах 855 и $893 \mathrm{~cm}^{-1}$ относятся к полносимметричным колебаниям $v_{s}$ и антисимметричным колебаниям $v_{a s}$ иона уранила соответственно. На рис. 3 видно, что в спектре КР дейтерированных ураниловых соединений наблюдается группа из четырех линий с частотами $2075-2245 \mathrm{~cm}^{-1}$, соответствующих осцилляциям дейтерия. В случае водородных связей (рис. 2) такие моды должны проявляться в более длинноволновой области спектра: около $3000 \mathrm{~cm}^{-1}$. Зафиксировать эти полосы на нашей установке не удалось, так как спектральный диапазон используемого миниспектрометра составлял $0-2550 \mathrm{~cm}^{-1}$.

\section{Заключение}

Таким образом, использование светосильного волоконно-оптического миниспектрометра с многоэлементным приемником излучения и с фокусировкой инфракрасного $(785 \mathrm{~nm})$ лазерного излучения в небольшом объеме вещества обеспечило возможность регистрации спектров КР в ураниловых соединениях при небольших экспозициях. При этом в спектрах КР всех исследованных ацетатах уранилов был зарегистрирован характерный спутник на частоте около $855 \mathrm{~cm}^{-1}$. Эта линия КР относится к полносимметричному валентному колебанию иона уранила $\mathrm{UO}_{2}$, что позволяет надежно устанавливать наличие ураниловых соединений в исследуемых объектах и в окружающей среде. Показано, что спектры КР дейтерированных уранилацетатов существенно отличаются в области высоких частот от спектров водородсодержащих уранилацетатов. В спектрах КР на частотах $2075-2245 \mathrm{~cm}^{-1}$ дейтерированных уранилацетатов обнаруживаются четыре линии, относящиеся к колебаниям дейтерия, в то время как в спектрах водородсодержащих уранилацетатов в этой области линии КР отсутствуют.

\section{Финансирование работы}

Работа выполнена за счет Российского научного фонда, проект № 19-12-00242.

\section{Конфликт интересов}

Авторы заявляют, что у них нет конфликта интересов.

\section{Список литературы}

[1] Rabinowitch E., Linn $R$. Belford Spectroscopy and Photochemistry of Uranyl Compounds. N.Y.: The Macmillan Company, 1964. 375 p.

[2] Nichols E.L., Howes H.L. Fluorescence of the Uranyl Salts. Carnegie Inst. Wash. Pub. 1919. 298 p.

[3] Dieke G.H., Duncan A.B.F. Spectroscopic Properties of Uranium Compounds. National Nuclear Energy Series, Div. III. 2. N.Y.: McGraw-Hill, 1949. 290 p.

[4] Xiaoping Sun, Derrick R.J., Kolling H.M. // Inorg. Chim. Acta. 2015. V. 435. P. 117-124.

[5] Barraclough C.G., Cockman R.W., O’Donnell T.A. // Inorg. Nucl. Chem. Lett. 1981. V. 17. P. 83-86.

[6] Garg C.L., Narasimham K.V. // Spectrochim. Acta A. 1971. V. 27. P. $863-875$.

[7] Volod'ko L.V., Komyak A.I., Sleptsov // Zhurnal Prikladnoi Spectroskopii. 1965. V. 3. N 1. P. 65-71. 
[8] Горелик В.С., Пятышев А.Ю., Крылов А.С. // ФТТ. 2016. T. 58. B. 1. C. 163-169; Gorelik V.S., Pyatyshev A.Yu., Krylov A.S. // Phys. Solid State 2016. V. 58. N 1. P. 170-176.

[9] Kurtz S.K., Purry T.T. // J. Appl. Phys. 1968. V. 39. P. 3798-3813.

[10] Горелик В.С., Свербиль П.П. // Неорг. матер. 2015. Т. 51. № 11. C. 1190-1197; Gorelik V.S., Sverbil' P.P. // Inorg. Mater. 2015. V. 51. N 11. P. 1104-1110.

[11] Frost R.L., Čejka J., Godwin A.A. // J. Raman Spectr. 2007. V. 38. P. 1609-1614.

[12] Bastians G., Crump W.P., Griffith R., Withnall A. // J. Raman Spectr. 2004. V. 35. P. 726-731.

[13] Quilès F., Burneau A. //Vibrational Spectroscopy. 1998. V. 18. P. 61-75.

[14] Boikov V.N., Krasovskii A.N., Umreiko D.S. // J. Appl. Spectrosc. 1982. V. 37. P. 1277.

[15] McGlynn S.P., Smith J.K., Neely W.C. // J. Chem. Phys. 1961. V. 35. P. 105-116.

[16] Володько Л.В., Комяк А.И., Умрейко Д.С. Ураниловые соединения (спектры, строение). Т. 1. Минск: Изд-во БГУ, 1981. $432 \mathrm{c}$

[17] Сережкина Л.Б., Вологжсанина А.В., Клепов В.В., Сережскин В.Н. // Кристаллография. 2010. Т. 55. № 5. С. 822 828.

[18] Кизель В.А., Красилов Ю.И., Бурков В.И. // УФН. 1974. T. 114. C. 295-349.

[19] Klepov V.V., Vologzhanina A.V., Alekseev E.V., Pushkin D.V., Serezhkina L.B., Sergeeva O.A., Knyazev A.V., Serezhkin V.N. // Cryst. Eng. Commun. 2016. V. 18. P. 1723-1731.

[20] Клепов В.В. Автореф. канд. дис. Самара: Самарский государственный университет. 2014. С. 165.

[21] Горелик В.С., Литвинова А.О., Умаров М.Ф. // Краткие сообщения по физике. ФИАН им. П.Н. Лебедева РАН. 2014. № 11. C. 3-9. 